Article

\title{
TSLS and LIML Estimators in Panels with Unobserved Shocks
}

\author{
Giovanni Forchini ${ }^{1, *(1)}$, Bin Jiang ${ }^{2}$ and Bin Peng ${ }^{3}$ \\ 1 Department of Economics, Umeå University, 90187 Umeå , Sweden \\ 2 Department of Econometrics and Business Statistics, Monash University, Clayton VIC 3800, Australia; \\ Bin.Jiang@monash.edu \\ 3 Department of Economics, University of Bath, Bath BA2 7AY, UK; b.peng2@bath.ac.uk \\ * Correspondence: Giovanni.Forchini@umu.se; Tel.: +46-90-7867876
}

Received: 17 August 2017; Accepted: 27 March 2018; Published: 9 April 2018

\begin{abstract}
The properties of the two stage least squares (TSLS) and limited information maximum likelihood (LIML) estimators in panel data models where the observables are affected by common shocks, modelled through unobservable factors, are studied for the case where the time series dimension is fixed. We show that the key assumption in determining the consistency of the panel TSLS and LIML estimators, as the cross section dimension tends to infinity, is the lack of correlation between the factor loadings in the errors and in the exogenous variables-including the instruments - conditional on the common shocks. If this condition fails, both estimators have degenerate distributions. When the panel TSLS and LIML estimators are consistent, they have covariance-matrix mixed-normal distributions asymptotically. Tests on the coefficients can be constructed in the usual way and have standard distributions under the null hypothesis.
\end{abstract}

Keywords: two-stage least squares; limited information maximum likelihood; common shocks

JEL Classification: C23; C26; C38

\section{Introduction}

Macroeconomic, technological, institutional, political, environmental, health, and sociological shocks are plausible in empirical research in many situations. For example, a researcher may be using a panel of countries to investigate a composite measure of health care attainment in terms of per capita health expenditure and educational attainment. Both the explained and the explanatory variables may be affected by the introduction of new medical technologies, medicines, hospital procedures, the occurrence of a flu epidemic, or of a particular cold winter or hot summer in a large region, or, as in recent years, the financial crisis. Such shocks are not observed. They affect several countries and their impact depends on the characteristics of the countries themselves. Similar problems arise in micro-econometric studies. For example, in analyzing a panel of executive compensation in terms of returns on assets, stock returns, level of responsibility and gender (among other explanatory variables), unobservable financial, political, environmental as well as industry specific shocks may occur and may affect both the dependent variables and the regressors simultaneously.

Andrews (2005) has shown that common shocks-modelled by unobservable common factors in both the disturbance and the regressors-strongly affect the properties of the ordinary least squares (OLS) estimator in a linear regression model. Precisely, Andrews (2005) shows that, in order for the OLS estimator to be consistent, the factor loadings for the disturbance and the regressors must be uncorrelated conditional on the unobservable factors. These results have profound implications for applied researchers since reliable inferences based on OLS in the presence of common shock require 
very strong assumptions. In panel data with both large time series and cross-sectional dimensions, the assumption that the factor loadings in the disturbances and the regressors are conditionally uncorrelated can be relaxed (e.g., Pesaran 2006; Bai 2009). However, this situation is certainly not the norm in microeconometric applications, where the time dimension tends to be limited.

Since the common shocks affect both the errors and the regressors, they induce correlation between some of the regressors and the disturbance term (we will refer to this as factors endogeneity). Econometric models often contain explanatory variables that are endogenous due to simultaneity so that the dependent variable and some of the explanatory variables are co-determined (we will refer to this as classical endogeneity). In the presence of instrumental variables, two standard approaches to endogeneity in panel data are the panel two-stage least squares (TSLS) (e.g., among others Wooldridge 2005; Arellano 2016) and the panel limited information maximum likelihood (LIML) estimators (e.g., Wansbeek and Meijer 2000; Alonso-Borrego and Arellano 1999; and Wansbeek and Prak 2017). This paper investigates how the panel TSLS and LIML estimators are affected by common shocks for which, surprisingly, no results seem available in the literature.

The literature on the effects of common shocks in models affected by classical endogeneity is very small. Ahn et al. $(2001,2013)$ generalize a fixed effects model in which the unobserved individual effects vary over time, and they propose a generalized method of moments (GMM) estimator that generalizes the fixed effects estimator through quasi-differencing. Robertson and Sarafidis (2015) consider linear panel data models with classical endogeneity in which the common factors affect the errors and the factor loading may be correlated with the exogenous variables. Following Ahn et al. (2001, 2013), Robertson and Sarafidis (2015) regard the common factors as unknown parameters, investigate the identification conditions and suggest a GMM estimator. Notice that this literature on the effects of common shocks differs from the one initiated by Andrews (2005) in two fundamental ways: (1) common factors are regarded as parameters not as random variables; (2) the explanatory variables are correlated to the error factor loadings while Andrews (2005) assumes that both the explanatory variables and the error term depend on the same factors.

Harding and Lamarche $(2011,2014)$ extend the model of Pesaran (2006) to allow for classical endogeneity. Precisely, Harding and Lamarche (2011) show that the estimators suggested by Pesaran (2006) also account for classical endogeneity when both the time series and the cross sectional dimensions are large (see also Harding and Lamarche (2014) for an approach based on quantiles). Thus, they investigate how the estimators of Pesaran (2006) are affected by classical endogeneity but are uninformative about how classical estimators are affected by factor endogeneity. Notice also that, by assuming that $(T, N)$ tends to infinity, one allows the information about the shocks to accumulate over time. This is not usually a reasonable assumption in micro-econometric studies where the time dimension tends to be small.

This paper investigates the effects of factor endogeneity on standard estimators used in the presence of classical endogeneity by studying the asymptotic properties of the panel TSLS and LIML estimators. Our results, which are in line with those of Andrews (2005), show that as the cross-sectional dimension tends to infinity (for a fixed time dimension):

1. the panel TSLS and LIML estimators have a non-degenerate non-standard asymptotic distribution if the factor loadings in the explanatory variables and the instruments are correlated to the reduced form errors conditional on the common factors; and

2. they are consistent but have mixed-normal asymptotic distributions when the factor loadings in the explanatory variables and the instruments are uncorrelated to the reduced form errors conditional on the common factors. In this case, tests on the structural coefficients can be constructed in the usual way and have standard distributions under the null hypothesis.

Therefore, the presence of common shocks may have a significant impact on the statistical properties of the TSLS and the LIML estimators depending on the properties of the errors and regressors conditional on the common shocks. These estimators are consistent if the reduced form errors and regressors (including the instruments) are conditionally independent given the common shocks, but 
they are inconsistent otherwise. In other words, consistency of the TSLS and LIML estimators holds if the the model satisfies classical conditions of the validity of instrumental variables estimators conditional on the shocks (i.e., the 'exogenous' variables must be uncorrelated with the errors given the shocks and the instruments need to be correlated with the right-hand-side endogenous variables but be uncorrelated with the errors conditional on the shocks). As far as we know, there are no tests for conditional independence of the factor loadings affecting the endogenous and the exogenous variables directly. Thus, this work draws the attention of researchers on the possible inferential problems affecting classical estimators when unobservable shocks are plausible.

The rest of the paper is organized as follows. Section 2 presents the model, the estimators considered, and the technical assumptions underlying the model. Section 3 contains the main results and Section 4 concludes. A discussion of some technical results-including stable convergence, conditional strong law of large numbers and conditional central limit theorem-and proofs of the main results are in an online supplementary file.

\section{The Model}

We consider a panel data structural equation model with a fixed number of time periods $T \geq 1$. The observations for unit $i$ concerning an endogenous variable observed over $T$ periods are collected in the $T \times 1$ vector $y_{1, i}$. This depends linearly on the endogenous variables in $y_{2, i}$, the exogenous variables in $z_{1, i}$, and an unobservable structural error $u_{1, i}$ :

$$
\underset{(T \times 1)}{y_{1, i}}=\underset{(T \times 1)}{\delta_{0}}+\underset{(T \times p)}{y_{2, i}} \underset{(p \times 1)}{\beta_{0}}+\underset{\left(T \times k_{1}\right)}{z_{1, i}} \underset{\left(k_{1} \times 1\right)}{\alpha_{0}}+\underset{(T \times 1)}{u_{1, i}},
$$

where $\delta_{0}, \beta_{0}$ and $\alpha_{0}$ are the structural parameters. The dimensions of vectors and matrices are reported in brackets the first time they are used, unless they are obvious from the context. The reduced form for $y_{2, i}$ is

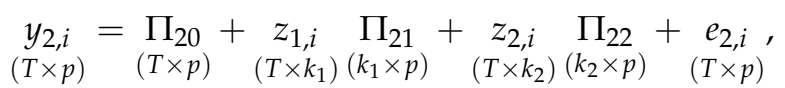

where $z_{2, i}$ denotes the observations on the exogenous variables excluded from the structural equation, usually referred to as the instruments, $e_{2, i}$ is unobservable reduced form errors, and $\Pi_{20}, \Pi_{21}$ and $\Pi_{22}$ are the reduced form parameters. Notice that, for $T=1$, Equations (1) and (2) form a classical cross-sectional structural equations model. We will now discuss how a factor structure can be introduced in both the errors and some of the regressors in Equation (1).

To allow for a general model, we define the two matrices $z_{1, i}=\left(\begin{array}{c}w_{1, i}, x_{1, i}\end{array}\right) \quad \begin{gathered}S_{1} \\ \left(\left(T \times h_{1}\right)\right.\end{gathered}$ and $z_{2, i}=\underset{\left(T \times h_{3}\right)}{\left(w_{2, i}, x_{2, i}\right)} \underset{\left(T \times h_{4}\right)}{{ }_{\left(\left(h_{3}+h_{4}\right) \times k_{2}\right)}}$, where $h_{1}+h_{2} \geqslant k_{1}, h_{3}+h_{4} \geqslant k_{2}$. The matrices $S_{1}$ and $S_{2}$ are fixed selection matrices of full rank equal to, respectively, $k_{1}$ and $k_{2}$. The matrices $w_{1, i}$ and $w_{2, i}$ represent observations on variables that are not affected by the common shocks such as gender, age and nationality; $x_{1, i}$ and $x_{2, i}$ contain observations on variables that are affected by common shocks.

We model common shocks by using unobservable factors structures. Precisely, we assume that

$$
x_{i}=\left(x_{1, i}, x_{2, i}\right)=F_{T} \Gamma_{i}+v_{i},
$$

where $F_{T}=\left(f_{1}{ }^{\prime}, \ldots, f_{T}{ }^{\prime}\right)^{\prime}, f_{t}$ for $t=1, \ldots, T$ is a $(1 \times m)$ unobservable vector of factors capturing the common shocks at time $t, \Gamma_{i}=\underset{\left(m \times h_{2}\right)}{\left(\Gamma_{1, i}, \Gamma_{2, i}\right)}$ is a random matrix of factor loadings, and $v_{i}=\left(v_{1, i}, v_{2, i}\right)$ is a random matrix representing the values of the exogenous variables $\left(x_{1, i}, x_{2, i}\right)$ $\left(T \times h_{2}\right)\left(T \times h_{4}\right)$

that one would observe if there were no common shocks. Notice that, although the shocks are common, 
the way they affect each unit $i$ is determined by $\Gamma_{i}$, which varies randomly from an individual to another. The number of factors, $m$, affecting the regressors is finite but unknown.

We also assume that the common shocks affect the error terms. Without loss of generality, we impose a factor structure on the reduced form errors and see how this implies a factor structure in the error of Equation (1). Let

$$
y_{i}=\left(y_{1, i}, y_{2, i}\right)=\left(\pi_{10}, \Pi_{20}\right)+z_{1, i}\left(\pi_{11}, \Pi_{21}\right)+z_{2, i}\left(\pi_{12}, \Pi_{22}\right)+\left(e_{1, i}, e_{2, i}\right)
$$

then, the reduced form errors satisfy

$$
e_{i}=\left(e_{1, i}, e_{2, i}\right)=F_{T} \gamma_{i}+\left(\varepsilon_{1, i}, \varepsilon_{2, i}\right)
$$

where $\gamma_{i}=\left(\gamma_{1, i}, \gamma_{2, i}\right)$ is a matrix of factor loadings and $\varepsilon_{i}=\left(\varepsilon_{1, i}, \varepsilon_{2, i}\right)$ are proper idiosyncratic $(m \times 1)(m \times p) \quad(T \times 1)(T \times p)$

errors. Once again, different units are affected by the common shocks in different ways because the factor loadings vary randomly among units. The model considered has classical endogeneity due to the presence of the endogenous variables on the right-hand side of the structural equation. It also has factors' endogeneity induced by the common shocks in the reduced form. To see this, we replace the reduced form into the structural equation, and obtain the compatibility restrictions

$$
\pi_{10}=\Pi_{20} \beta_{0}+\delta_{0}, \quad \pi_{11}=\Pi_{21} \beta_{0}+\alpha_{0}, \quad \pi_{12}=\Pi_{22} \beta_{0},
$$

and

$$
e_{1, i}=u_{1, i}+e_{2, i} \beta_{0}
$$

It follows from Equation (6) that the structural parameter $\beta_{0}$ is identified if and only if

1. $\left(\pi_{12}, \Pi_{22}\right)$ is identified, and

2. $\operatorname{rank}\left(\Pi_{22}\right)=p \leqslant k_{2}$.

In order to identify $\alpha_{0}$ and $\delta_{0}$, one also needs $\left(\pi_{11}, \Pi_{21}\right)$ and $\left(\pi_{10}, \Pi_{21}\right)$ to be identified. Moreover, rewriting Equation (7) using Equations (4) and (5), we obtain

$$
u_{1, i}=e_{1, i}-e_{2, i} \beta_{0}=F_{T}\left(\gamma_{1 i}-\gamma_{2 i} \beta_{0}\right)+\varepsilon_{1, i}-\varepsilon_{2, i} \beta_{0}
$$

Thus, the structural error also has a factor structure with factor loadings $\gamma_{1 i}-\gamma_{2 i} \beta_{0}$.

The model considered allows for the case in which the common factors affect the errors (both in the reduced form and the structural equation) as well as some of the instruments. It therefore entails a complex interaction between factors and classical endogeneity. Notice that the model could be generalized by imposing a more complex structure on the error term $\left(\varepsilon_{1, i}, \varepsilon_{2, i}\right)$ to allow for unobserved individual effects as in a random or fixed effects model.

We now briefly discuss the main differences between the model considered here and the models of Robertson and Sarafidis (2015) and Harding and Lamarche (2011). Robertson and Sarafidis (2015) regard the factors as unknown parameters. As a consequence, they require some extra identification conditions and standardizations, which include, for example, bounds on the number of factors that depend on the time series dimension of the panel (e.g., to have $m=2$ factors the the number of waves must be $T \geq 5$ ). A second difference is related to the specification of our Equation (3), which allows the common shocks to affect the exogenous variables as additive shocks that may induce correlation between exogenous variables and the error terms through the correlation between $\Gamma_{i}$ and $\gamma_{i}$. On the other hand, Robertson and Sarafidis (2015) allow for a correlation between $v_{i}$ and $\gamma_{i}$ but assume the factors to be constant parameters.

Harding and Lamarche (2011) allow the reduced form errors to have a factor structure (so that both $u_{1, i}$ and $y_{2, i}$ have a factor structure) with unobserved but random factors but the instruments $z_{2, i}$ 
are assumed not to be affected by shocks. They also focus on the case where $T$ and $N$ are large and study the properties of the CCEP and CCEMG of Pesaran (2006) but not those of standard estimators.

\subsection{TSLS and LIML Estimators}

$$
\begin{gathered}
\text { Let } z_{i}=\left(z_{1, i}, z_{2, i}\right) \text { and } \Pi=\left(\begin{array}{cc}
\pi_{11} & \Pi_{21} \\
\pi_{12} & \Pi_{22}
\end{array}\right) . \text { The reduced form can then be written as } \\
y_{i}=\left(\pi_{10}, \Pi_{20}\right)+z_{i} \Pi+e_{i},
\end{gathered}
$$

and the OLS estimators of $\Pi$ and $\left(\pi_{10}, \Pi_{20}\right)$ are respectively

$$
\hat{\Pi}=\left(\begin{array}{ll}
\hat{\pi}_{11} & \hat{\Pi}_{21} \\
\hat{\pi}_{12} & \hat{\Pi}_{22}
\end{array}\right)=\left(\frac{1}{N} \sum_{i=1}^{N} z_{i}^{\prime} z_{i}-\bar{z}^{\prime} \bar{z}\right)^{-1}\left(\frac{1}{N} \sum_{i=1}^{N} z_{i}^{\prime} y_{i}-\bar{z}^{\prime} \bar{y}\right)
$$

and

$$
\left(\hat{\pi}_{10}, \hat{\Pi}_{20}\right)=\bar{y}-\bar{z} \hat{\Pi},
$$

where $\bar{y}=\frac{1}{N} \sum_{i=1}^{N} y_{i}$, and $\bar{z}=\frac{1}{N} \sum_{i=1}^{N} z_{i}$.

Let

$$
\begin{aligned}
& \hat{H}=\frac{1}{N}\left(\sum_{i=1}^{N} \tilde{z}_{2, i}^{\prime} \tilde{z}_{2, i}-\sum_{i=1}^{N} \tilde{z}_{2, i}^{\prime} \tilde{z}_{1, i}\left(\sum_{i=1}^{N} \tilde{z}_{1, i}^{\prime} \tilde{z}_{1, i}\right)^{-1} \sum_{i=1}^{N} \tilde{z}_{1, i}^{\prime} \tilde{z}_{2, i}\right), \\
& \hat{\Omega}=\frac{1}{N}\left(\sum_{i=1}^{N} \tilde{y}_{i}^{\prime} \tilde{y}_{i}-\sum_{i=1}^{N} \tilde{y}_{i}^{\prime} \tilde{z}_{i}\left(\sum_{i=1}^{N} \tilde{z}_{i}^{\prime} \tilde{z}_{i}\right)^{-1} \sum_{i=1}^{N} \tilde{z}_{i}^{\prime} \tilde{y}_{i}\right),
\end{aligned}
$$

where $\tilde{y}_{i}=y_{i}-\bar{y}$, and $\tilde{z}_{1, i}, \tilde{z}_{2, i}$, and $\tilde{z}_{i}$ are defined similarly. Then, the panel TSLS estimator of $\beta$ is

$$
\hat{\beta}_{T S L S}=\left(\hat{\Pi}_{22}^{\prime} \hat{H} \hat{\Pi}_{22}\right)^{-1}\left(\hat{\Pi}_{22}^{\prime} \hat{H} \hat{\pi}_{12}\right)
$$

and the panel LIML estimator is

$$
\hat{\beta}_{\text {LIML }}=\underset{b}{\arg \min } \frac{\left(1,-b^{\prime}\right)\left(\hat{\pi}_{12}, \hat{\Pi}_{22}\right)^{\prime} \hat{H}\left(\hat{\pi}_{12}, \hat{\Pi}_{22}\right)\left(1,-b^{\prime}\right)^{\prime}}{\left(1,-b^{\prime}\right) \hat{\Omega}\left(1,-b^{\prime}\right)^{\prime}} .
$$

The estimators for $\alpha_{0}$ and $\delta_{0}$ are respectively

$$
\hat{\alpha}=\hat{\pi}_{11}-\hat{\Pi}_{21} \hat{\beta}, \quad \hat{\delta}=\hat{\pi}_{10}-\hat{\Pi}_{20} \hat{\beta},
$$

where $\hat{\beta}$ can be either the panel TSLS or LIML estimator. Notice that the panel TSLS and LIML estimators of the structural coefficients reduce to the classical TSLS and LIML estimators when $T=1$.

\subsection{Model Assumptions}

Following Andrews (2005) and Kuersteiner and Prucha (2013), it is natural to state the assumptions for our model conditional on the factors. In order to do this, we will regard all variables as defined on a probability space $(\Omega, \mathcal{A}, P)$. The sigma-algebra generated by the random vector vec $\left(F_{T}\right)$ is denoted by $\mathcal{F}=\left\{\omega \in \mathcal{A}: \operatorname{vec}\left(F_{T}\right)(\omega) \in \mathbb{B}^{T m}\right\}$, where $\mathbb{B}^{T m}$ is the Borel sigma algebra in $\mathbb{R}^{T m}$. In addition, in the rest of this paper, $\|A\|_{2}$ denotes $\sqrt{\operatorname{tr}\left(A^{\prime} A\right)}$, where the operator $\operatorname{tr}(\cdot)$ is the trace of a square matrix.

The assumptions of the model are formulated conditional on $\mathcal{F}$ to allow for the application of a conditional version of the strong law of large numbers (e.g., Majerek et al. 2005; Rao 2009; and 
Cabrera et al. 2012) and the central limit theorem (e.g., Dedecker and Merlevede 2002; Grzenda and Zięba 2008; and Yuan et al. 2014) in the hope to make the paper accessible to practitioners. The results could be obtained under more general conditions along the lines of the work of Kuersteiner and Prucha (2013), which allows for sequential exogeneity. Notice, however, that the main condition for consistency of the TSLS and LIML estimators that we identify is the lack of correlation between the factor loadings in the exogenous variables and those in the errors conditional on the unobservable factors. Even if all other assumptions are weakened, this condition cannot be relaxed.

For notational simplicity, we define the following $\mathcal{F}$-measurable matrices:

$$
\begin{aligned}
Z\left(F_{T}\right) & =\left(\begin{array}{ll}
Z_{11}\left(F_{T}\right) & Z_{12}\left(F_{T}\right) \\
Z_{21}\left(F_{T}\right) & Z_{22}\left(F_{T}\right)
\end{array}\right), \quad Z_{j, k}\left(F_{T}\right)=\lim _{N \rightarrow \infty} \frac{1}{N} \sum_{i=1}^{N} E\left[z_{j, i}{ }^{\prime} z_{k, i} \mid \mathcal{F}\right] \text { for } j, k=1,2, \\
Z^{*}\left(F_{T}\right) & =\left(\begin{array}{ll}
Z_{11}^{*}\left(F_{T}\right) & Z_{12}^{*}\left(F_{T}\right) \\
Z_{21}^{*}\left(F_{T}\right) & Z_{22}^{*}\left(F_{T}\right)
\end{array}\right)=Z\left(F_{T}\right)-z\left(F_{T}\right)^{\prime} z\left(F_{T}\right),
\end{aligned}
$$

where $z\left(F_{T}\right)=\lim _{N \rightarrow \infty} \frac{1}{N} \sum_{i=1}^{N} E\left[\left(z_{1, i}, z_{2, i}\right) \mid \mathcal{F}\right]$. We make the following two sets of assumptions.

Assumption 1. Let $\Delta$ be arbitrary $\mathcal{F}$-measurable, and $\Delta<\infty$ a.s. and let $\epsilon>0$.

$i \quad$ The random matrices $\varepsilon_{i}=\left(\varepsilon_{1, i}, \varepsilon_{2, i}\right)$ for $i=1,2, \ldots, N$ form a sequence of $\mathcal{F}$-independent random matrices, and have mean 0 and $E\left[\left\|\varepsilon_{i}\right\|_{2}{ }^{2+\epsilon} \mid \mathcal{F}\right]<\Delta$ a.s. over $i$ for some $\epsilon>0$. Moreover, $\Sigma_{\varepsilon}\left(F_{T}\right)=\lim _{N \rightarrow \infty} \frac{1}{N} \sum_{i=1}^{N} E\left[\varepsilon_{i}{ }^{\prime} \varepsilon_{i} \mid \mathcal{F}\right]$, where $\Sigma_{\varepsilon}\left(F_{T}\right)$ is $\mathcal{F}$-measurable.

ii $\quad$ The random matrices $v_{i}=\left(v_{1, i}, v_{2, i}\right)$ for $i=1,2, \ldots, N$ form a sequence of $\mathcal{F}$-independent random matrices, $E\left[\left\|v_{i}\right\|_{2}{ }^{4+\epsilon} \mid \mathcal{F}\right]<\Delta$ a.s. over i for some $\epsilon>0$. Moreover, $v\left(F_{T}\right)=\lim _{N \rightarrow \infty} \frac{1}{N} \sum_{i=1}^{N} E\left[v_{i} \mid \mathcal{F}\right]$ and $V\left(F_{T}\right)=\lim _{N \rightarrow \infty} \frac{1}{N} \sum_{i=1}^{N} E\left[v_{i}{ }^{\prime} v_{i} \mid \mathcal{F}\right]$, where $v\left(F_{T}\right)$ and $V\left(F_{T}\right)$ are $\mathcal{F}$-measurable.

iii The random matrices $w_{i}=\left(w_{1, i}, w_{2, i}\right)$ for $i=1,2, \ldots, N$ form a sequence of $\mathcal{F}$-independent random matrices with $E\left[\left\|w_{i}\right\|_{2}{ }^{4+\epsilon} \mid \mathcal{F}\right]<\Delta$ a.s. over $i$ for some $\epsilon>0$. Moreover, $w\left(F_{T}\right)=\lim _{N \rightarrow \infty} \frac{1}{N} \sum_{i=1}^{N} E\left[w_{i} \mid \mathcal{F}\right]$ and $W\left(F_{T}\right)=\lim _{N \rightarrow \infty} \frac{1}{N} \sum_{i=1}^{N} E\left[w_{i}{ }^{\prime} w_{i} \mid \mathcal{F}\right]$, where $w\left(F_{T}\right)$ and $W\left(F_{T}\right)$ are $\mathcal{F}$-measurable.

iv $\quad$ The factor loadings $\gamma_{i}=\left(\gamma_{i 1}, \gamma_{i 2}\right)$ for $i=1,2, \ldots, N$ form a sequence of $\mathcal{F}$-independent random matrices with mean $E\left[\gamma_{i} \mid \mathcal{F}\right]=\gamma\left(F_{T}\right)$ and $E\left[\left\|\gamma_{i}\right\|_{2}{ }^{2+\epsilon} \mid \mathcal{F}\right]<\Delta$ a.s. over i for some $\epsilon>0$, where $\gamma\left(F_{T}\right)$ is $\mathcal{F}$-measurable.

$v \quad$ The factor loadings $\Gamma_{i}$ for $i=1,2, \ldots, N$ form a sequence of $\mathcal{F}$-independent random matrices with $E\left[\Gamma_{i} \mid \mathcal{F}\right]=\Gamma\left(F_{T}\right)$ and $E\left[\left\|\Gamma_{i}\right\|_{2}^{4+\epsilon} \mid \mathcal{F}\right]<\Delta$ a.s. over i for some $\epsilon>0$.

vi The random matrices $w_{i}, v_{i}, \varepsilon_{i}, \Gamma_{i}$ and $\gamma_{i}$ are uncorrelated conditional on $\mathcal{F}$. $\left\|F_{T}\right\|_{2}<\infty$ a.s.

vii The random matrices $w_{i}, v_{i}, \varepsilon_{i}$ and $\left(\Gamma_{i}, \gamma_{i}\right)$ are uncorrelated conditional on $\mathcal{F}$. $\left\|F_{T}\right\|_{2}<\infty$ a.s.

Reviews and discussions of the concept of conditional independence are given by Phillips (1988), Majerek et al. (2005), Rao (2009) and Roussas (2008). Notice that conditional independence does not imply unconditional independence (see Phillips 1988 for conditions under which this is the case). Since $\gamma_{i}, \Gamma_{i}, w_{i}, v_{i}$ and $\varepsilon_{i}$ are heterogeneous conditional on $\mathcal{F}$, they are not only unconditionally dependent but also non-identically distributed. This is an extension of Andrews (2005) whose assumptions imply that such quantities are exchangeable and hence identically distributed. Appendix A in the online Supplementary Material contains a brief review of the various technical concepts used and gives further references. 
Assumption 1 implies that the reduced form error $\varepsilon_{i}$ and the exogenous variable $z_{i}$ are uncorrelated conditional on $\mathcal{F}$. Notice however that our model allows $\gamma_{i}, \Gamma_{i}, w_{i}, v_{i}$ and $\varepsilon_{i}$ to be (unconditionally) dependent among themselves and/or over units (e.g., Phillips 1988). This assumption could have been formulated in term of the structural equation and the reduced form for $y_{2, i}$ in an equivalent way given the one-to-one connection between structural and reduced form.

The conditional heterogeneity allowed by Assumption 1 implies that the shocks affect each individual differently through the terms $F_{T} \Gamma_{i}$ and $F_{T} \gamma_{i}$ as well as through the conditional means $E\left[v_{i} \mid \mathcal{F}\right]$ and $E\left[w_{i} \mid \mathcal{F}\right]$ and the error covariance matrix $E\left[\varepsilon_{i}^{\prime} \varepsilon_{i} \mid \mathcal{F}\right]$.

In a standard set-up, where there are no common shocks, it is possible to establish the identification of the structural parameters in terms of the reduced form parameters since the latter are identified and can be consistently estimated. It will be shown in Section 3 that if Assumption $1 \mathrm{i}-\mathrm{vi}$ holds, $\hat{\Pi}$ is a consistent estimator of $\Pi$ as $N \rightarrow \infty$. Hence, in this case, one can state the identification conditions for the structural parameters $\beta_{0}$ and $\alpha_{0}$ as follows.

\section{Assumption 2.}

$i \quad \operatorname{rank}\left(\Pi_{22}\right)=p \leqslant k_{2}$ and is satisfied.

ii $\quad Z^{*}\left(F_{T}\right)$ is non-singular and $H\left(F_{T}\right)=Z_{22}^{*}\left(F_{T}\right)-Z_{21}^{*}\left(F_{T}\right) Z_{11}^{*}\left(F_{T}\right)^{-1} Z_{12}^{*}\left(F_{T}\right)$ is positive definite uniformly.

Together with Assumption 1 i-vi, Assumption 2 provides identification restrictions, which are analogous to those for structural equations in the classical set-up (e.g., Assumptions 2.2 and 2.3 of Hausman (1983, p. 398), and Schmidt (1976, chp. 4)). Notice that Assumption 2 on its own does not identify $\beta_{0}$ or $\alpha_{0}$ because $\Pi$ may itself be unidentified. This is the case, for example, if the factor loadings $\Gamma_{i}$ and $\gamma_{i}$ are not uncorrelated conditional on $\mathcal{F}$ : the reduced form errors and (some of) the reduced form regressors are correlated even conditional on the factors.

\section{Consistency and Asymptotic Distribution}

We now investigate the effects of common shocks on the panel TSLS and LIML estimators of the structural parameters. Notice that the presence of the factors in the reduced form errors and some of the regressors implies a correlation between the reduced form errors and regressors, so that the OLS estimator of the reduced form parameter, $\Pi$, may or may not be consistent. As a consequence, the panel TSLS and LIML estimators of the structural parameters may or may not be consistent.

We show that the panel LIML and TSLS estimators are consistent estimators if the factor loadings are uncorrelated conditional on $\mathcal{F}$. t. Let $w_{i}=\left(w_{1, i}, w_{2, i}\right)$ and $x_{i}=\left(x_{1, i}, x_{2, i}\right)$. Partition the selection matrices $\underset{\left(h_{1}+h_{2}\right) \times k_{1}}{S_{1}}=\left(\begin{array}{c}S_{11} \\ S_{12}\end{array}\right)$ and $\underset{\left(h_{3}+h_{4}\right) \times k_{2}}{S_{2}}=\left(\begin{array}{c}S_{21} \\ S_{22}\end{array}\right)$ conformably to $w_{i}$ and $x_{i}$, respectively, and define

$$
S=\left(\begin{array}{cc}
S_{11} & 0 \\
0 & S_{21} \\
S_{12} & 0 \\
0 & S_{22}
\end{array}\right)=\left(\underline{S}_{1}, \underline{S}_{2}\right)
$$

Notice that $z_{i}=\left(w_{i}, x_{i}\right) S, z_{i}^{\prime} z_{i}=S^{\prime}\left(\begin{array}{cc}w_{i}^{\prime} w_{i} & w_{i}^{\prime} x_{i} \\ x_{i}^{\prime} w_{i} & x_{i}^{\prime} x_{i}\end{array}\right) S$ and $z_{i}-\bar{z}=\left(\left(w_{i}, x_{i}\right)-(\bar{w}, \bar{x})\right) S$.

Lemma 1. Under Assumptions $1 i-v i$, as $N \rightarrow \infty, \hat{\Pi}-\Pi \rightarrow 0$ a.s. conditional on $\mathcal{F}$. Moreover, conditional on $\mathcal{F}$

$$
\sqrt{N} \operatorname{vec}(\hat{\Pi}-\Pi) \rightarrow^{D}\left(I_{p+1} \otimes\left(S^{\prime} Q^{*}\left(F_{T}\right) S\right)^{-1} S^{\prime}\right)\left(\Theta\left(F_{T}\right)\right)^{\frac{1}{2}} N\left(0, I_{\left(h_{1}+h_{2}+h_{3}+h_{4}\right)(p+1)}\right)
$$


where

$$
\begin{aligned}
Q^{*}\left(F_{T}\right)= & \left(\begin{array}{cc}
W^{*}\left(F_{T}\right) & W X^{*}\left(F_{T}\right) \\
W X^{*}\left(F_{T}\right)^{\prime} & X^{*}\left(F_{T}\right)
\end{array}\right), \\
W^{*}\left(F_{T}\right)= & W\left(F_{T}\right)-w\left(F_{T}\right)^{\prime} w\left(F_{T}\right), \\
X^{*}\left(F_{T}\right)= & V\left(F_{T}\right)-v\left(F_{T}\right)^{\prime} v\left(F_{T}\right) \\
& +\lim _{N \rightarrow \infty} \frac{1}{N} \sum_{i=1}^{N} E\left[\Gamma_{i}^{\prime} F_{T}^{\prime} F_{T} \Gamma_{i} \mid \mathcal{F}\right]-\Gamma\left(F_{T}\right)^{\prime} F_{T}^{\prime} F_{T} \Gamma\left(F_{T}\right), \\
W X^{*}\left(F_{T}\right)= & \lim _{N \rightarrow \infty} \frac{1}{N} \sum_{i=1}^{N} E\left[w_{i}^{\prime} v_{i} \mid \mathcal{F}\right]-w\left(F_{T}\right)^{\prime} v\left(F_{T}\right), \\
\Theta\left(F_{T}\right)= & \lim _{N \rightarrow \infty} \frac{1}{N} \sum_{i=1}^{N} \operatorname{cov}\left[\operatorname{vec}\left(\mathcal{E}_{i}\left(F_{T}\right)\right) \mid \mathcal{F}\right],
\end{aligned}
$$

and

$$
\mathcal{E}_{i}\left(F_{T}\right)=\left(\begin{array}{c}
w_{i}^{\prime}-\frac{1}{N} \sum_{j=1}^{N} E\left[w_{j}^{\prime} \mid \mathcal{F}\right] \\
v_{i}^{\prime}-\frac{1}{N} \sum_{j=1}^{N} E\left[v_{j}^{\prime} \mid \mathcal{F}\right]+\left(\Gamma_{i}-\Gamma\left(F_{T}\right)\right)^{\prime} F_{T}^{\prime}
\end{array}\right)\left(\varepsilon_{i}+F_{T}\left(\gamma_{i}-\gamma\left(F_{T}\right)\right)\right)
$$

Lemma 1 implies that $\hat{\Pi}$ is consistent and $\sqrt{N}$ vec $(\hat{\Pi}-\Pi)$ converges $\mathcal{F}$-stably to a random vector having a covariance matrix mixed-normal distribution (for the notion of stable convergence see Appendix A in the online Supplementary Material and references therein).

It follows from Lemma 1 that $\left(\hat{\pi}_{10}, \hat{\Pi}_{20}\right)=\left(\pi_{10}, \Pi_{20}\right)-\bar{z}(\hat{\Pi}-\Pi)+\bar{e}$ so that $\left(\hat{\pi}_{10}, \hat{\Pi}_{20}\right) \rightarrow$ $\left(\pi_{10}, \Pi_{20}\right)$ a.s. if and only if $\bar{e} \rightarrow 0$ a.s., which requires $\gamma\left(F_{T}\right)=0$ a.s. The inconsistency of the OLS estimator of the constant has been noted by Andrews (2005) for the case of i.i.d. observations conditional on $\mathcal{F}$.

If the factor loadings in the reduced form explanatory variables and the reduced form errors are not independent conditional on $\mathcal{F}$, then the reduced form parameters cannot be estimated consistently using OLS. This is the case because conditioning on the shocks does not remove the correlation between the reduced form errors and explanatory variables.

Lemma 2. Under Assumptions $1 i-v$ and vii, then, as $N \rightarrow \infty$,

$$
\hat{\Pi}=\Pi+\Delta\left(F_{T}\right) \quad \text { a.s., }
$$

where $\Delta\left(F_{T}\right)=\left(S^{\prime} Q^{*}\left(F_{T}\right) S\right)^{-1} S^{\prime} M\left(F_{T}\right), Q^{*}\left(F_{T}\right)$ is defined in Equation (17) and

$$
M\left(F_{T}\right)=\left(\begin{array}{c}
0 \\
\lim _{N \rightarrow \infty} \frac{1}{N} \sum_{i=1}^{N} E\left[\Gamma_{i}^{\prime} F_{T}^{\prime} F_{T} \gamma_{i} \mid \mathcal{F}\right]-\Gamma\left(F_{T}\right)^{\prime} F_{T}^{\prime} F_{T} \gamma\left(F_{T}\right)
\end{array}\right)
$$

Notice that if the factor loadings in the reduced form explanatory variables and the reduced form errors are correlated conditional on $\mathcal{F}$, the OLS estimator of the reduced form parameters has a non-degenerate asymptotic distribution as the cross-sectional dimension tends to infinity (cf. Theorem 1 of Andrews 2005). 
All results to follow depend on Lemmas 1 and 2. In this paper, these have been derived under Assumption 1. However, Equations (16) and (23) may hold under weaker conditions. Precisely, writing

$$
\begin{aligned}
\sqrt{N} \operatorname{vec}(\hat{\Pi}-\Pi)=\left(I_{p+1} \otimes\right. & \left.\left(\frac{1}{N} \sum_{i=1}^{N} S^{\prime}\left(\begin{array}{cc}
\left(w_{i}-\bar{w}\right)^{\prime}\left(w_{i}-\bar{w}\right) & \left(w_{i}-\bar{w}\right)^{\prime}\left(x_{i}-\bar{x}\right) \\
\left(x_{i}-\bar{x}\right)^{\prime}\left(w_{i}-\bar{w}\right) & \left(x_{i}-\bar{x}\right)^{\prime}\left(x_{i}-\bar{x}\right)
\end{array}\right)\right)^{-1} S^{\prime}\right) \times \\
& \frac{1}{\sqrt{N}} \sum_{i=1}^{N} \operatorname{vec}\left(\begin{array}{c}
\left(w_{i}-\bar{w}\right)^{\prime}\left(e_{i}-\bar{e}\right) \\
\left(x_{i}-\bar{x}\right)^{\prime}\left(e_{i}-\bar{e}\right)
\end{array}\right),
\end{aligned}
$$

we see that for Lemma 1 to hold one needs:

$$
\overline{1} \sum_{i=1}^{N}\left(\begin{array}{cc}
\left(w_{i}-\bar{w}\right)^{\prime}\left(w_{i}-\bar{w}\right) & \left(w_{i}-\bar{w}\right)^{\prime}\left(x_{i}-\bar{x}\right) \\
\left(x_{i}-\bar{x}\right)^{\prime}\left(w_{i}-\bar{w}\right) & \left(x_{i}-\bar{x}\right)^{\prime}\left(x_{i}-\bar{x}\right)
\end{array}\right) \rightarrow Q^{*}\left(F_{T}\right) \text { a.s., }
$$

and

$$
\frac{1}{\sqrt{N}} \sum_{i=1}^{N} \operatorname{vec}\left(\left(\begin{array}{c}
\left(w_{i}-\bar{w}\right)^{\prime} \\
\left(x_{i}-\bar{x}\right)^{\prime}
\end{array}\right)\left(e_{i}-\bar{e}\right)\right) \rightarrow^{D}\left(\Theta\left(F_{T}\right)\right)^{\frac{1}{2}} N\left(0, I_{\left(h_{1}+h_{2}+h_{3}+h_{4}\right)(p+1)}\right)
$$

$\mathcal{F}$-stably. These conditions can hold under weaker assumptions than the one used in this paper (cf. Kuersteiner and Prucha 2013), but would be less accessible to practitioners.

We focus on the coefficients of the endogenous variables.

Theorem 1. Under Assumptions $1 i-v i$ and 2, conditional on $\mathcal{F}$, as $N$ tends to infinity,

1. $\hat{\beta}_{T S L S} \rightarrow \beta_{0}$ a.s. and $\sqrt{N}\left(\hat{\beta}_{T S L S}-\beta_{0}\right) \rightarrow^{D} A\left(F_{T}\right) N\left(0, I_{\left(h_{1}+h_{2}+h_{3}+h_{4}\right)(p+1)}\right)$,

2. $\hat{\beta}_{\text {LIML }} \rightarrow \beta_{0}$ a.s. and $\sqrt{N}\left(\hat{\beta}_{\text {LIML }}-\beta_{0}\right) \rightarrow^{D} A\left(F_{T}\right) N\left(0, I_{\left(h_{1}+h_{2}+h_{3}+h_{4}\right)(p+1)}\right)$, where $A\left(F_{T}\right)$ is

$$
A\left(F_{T}\right)=\left(\Pi_{22}^{\prime} H\left(F_{T}\right) \Pi_{22}\right)^{-1} \Pi_{22}{ }^{\prime} H\left(F_{T}\right)\left(\left(1,-\beta_{0}{ }^{\prime}\right) \otimes\left(\left(0, I_{k_{2}}\right)\left(S^{\prime} Q^{*}\left(F_{T}\right) S\right)^{-1}\right) S^{\prime}\right)\left(\Theta\left(F_{T}\right)\right)^{\frac{1}{2}} .
$$

When the factor loadings in the reduced form explanatory variables and the reduced form errors are uncorrelated conditional on $\mathcal{F}$, the panel TSLS and LIML estimators are consistent and asymptotically equivalent in the sense that, once normalized, they converge $\mathcal{F}$-stably to the same covariance matrix mixed normal distribution. We will see in the next section that this allows the construction of standard tests on these coefficients. However, if $\gamma_{i}$ and $\Gamma_{i}$ are correlated conditional on $\mathcal{F}$, consistency does not hold.

Theorem 2. Suppose Assumption $1 i-v$ and vii hold. Conditional on $\mathcal{F}$, as $N \rightarrow \infty$,

1. The panel TSLS estimator has a non-degenerate asymptotic distribution

$$
\hat{\beta}_{\text {TSLS }} \rightarrow \beta_{0}+b\left(F_{T}\right) \text { a.s. }
$$

2. The panel LIML estimator has also a non-degenerate asymptotic distribution

$$
\hat{\beta}_{\text {LIML }} \rightarrow \beta_{0}+b\left(F_{T}\right) \text { a.s., }
$$

where $\Delta\left(F_{T}\right)$ is partitioned conformably to $\Pi$ as $\Delta\left(F_{T}\right)=\left(\begin{array}{cc}\Delta_{11}\left(F_{T}\right) & \Delta_{21}\left(F_{T}\right) \\ \Delta_{12}\left(F_{T}\right) & \Delta_{22}\left(F_{T}\right)\end{array}\right)$, and

$$
\begin{aligned}
b\left(F_{T}\right)= & {\left[\left(\Pi_{22}+\Delta_{22}\left(F_{T}\right)\right)^{\prime} H\left(F_{T}\right)\left(\Pi_{22}+\Delta_{22}\left(F_{T}\right)\right)\right]^{-1} } \\
& \times\left(\Pi_{22}+\Delta_{22}\left(F_{T}\right)\right)^{\prime} H\left(F_{T}\right)\left(\Delta_{12}\left(F_{T}\right)-\Delta_{22}\left(F_{T}\right) \beta_{0}\right) .
\end{aligned}
$$


Therefore, if the factor loadings in the reduced form explanatory variables and the reduced form errors are not uncorrelated conditional on $\mathcal{F}$, the panel TSLS and LIML estimators have the same asymptotic non-degenerate distribution. The fact that they have degenerate distribution should be expected from the result of Phillips (1988) in the presence of total lack of identification. Notice, however, that, in our case, TSLS and LIML have the same asymptotic distribution because the failure of identification of the structural parameters is due to failure of identification of the reduced form parameters not to failure of the rank condition.

We now focus on the coefficients of the exogenous variables in the structural equation.

Theorem 3. Under Assumptions $1 i-v i$ and 2, conditional on $\mathcal{F}$, as $N$ tends to infinity

1. $\hat{\alpha}_{T S L S} \rightarrow \alpha_{0}$ a.s. and $\sqrt{N}\left(\hat{\alpha}_{T S L S}-\alpha_{0}\right) \rightarrow^{D} A^{*}\left(F_{T}\right) N\left(0, I_{\left(h_{1}+h_{2}+h_{3}+h_{4}\right)(p+1)}\right)$,

2. $\hat{\alpha}_{L I M L} \rightarrow \alpha_{0}$ a.s. and $\sqrt{N}\left(\hat{\alpha}_{L I M L}-\alpha_{0}\right) \rightarrow^{D} A^{*}\left(F_{T}\right) N\left(0, I_{\left(h_{1}+h_{2}+h_{3}+h_{4}\right)(p+1)}\right)$,

where

$$
\begin{aligned}
A^{*}\left(F_{T}\right) & =\left(1,-\beta_{0}^{\prime}\right) \otimes \\
& \left(\left(\left(I_{k_{1}}, 0\right)-\Pi_{21}\left(\Pi_{22}^{\prime} H\left(F_{T}\right) \Pi_{22}\right)^{-1} \Pi_{22}{ }^{\prime} H\left(F_{T}\right)\left(0, I_{k_{2}}\right)\right)\left(S^{\prime} Q^{*}\left(F_{T}\right) S\right)^{-1} S^{\prime}\right) \Theta\left(F_{T}\right)^{\frac{1}{2}} .
\end{aligned}
$$

Moreover, if we let $\gamma\left(F_{T}\right)=\underset{(m \times 1)}{\left(\underset{1}{(}\left(F_{T}\right)\right.} \underset{m \times p}{\left.\gamma_{2}\left(F_{T}\right)\right)}$, then

3. $\hat{\delta}_{T S L S} \rightarrow \delta_{0}+F_{T} \gamma_{1}\left(F_{T}\right)-F_{T} \gamma_{2}\left(F_{T}\right) \beta_{0}$ a.s.

4. $\quad \hat{\delta}_{\text {LIML }} \rightarrow \delta_{0}+F_{T} \gamma_{1}\left(F_{T}\right)-F_{T} \gamma_{2}\left(F_{T}\right) \beta_{0}$ a.s.

If the factor loadings in the reduced form explanatory variables and the reduced form errors are uncorrelated conditional on $\mathcal{F}$, the panel TSLS and LIML estimators of the coefficients of the exogenous variables in the structural equation are consistent and have a covariance matrix mixed normal distribution. The estimators of the constant, however, have a non-degenerate distribution unless $\gamma\left(F_{T}\right)=0$ a.e.

If the factor loadings in the reduced form explanatory variables and the reduced form errors are not uncorrelated conditional on $\mathcal{F}$, the panel TSLS and LIML estimators for $\alpha_{0}$ have the same non-degenerated distribution as the following result shows.

Theorem 4. Under Assumptions $1 i-v$, vii and 2, conditional on $\mathcal{F}$, as $N$ tends to infinity

1. $\hat{\alpha}_{T S L S} \rightarrow \alpha_{0}+\Delta_{11}\left(F_{T}\right)+\Pi_{21} b\left(F_{T}\right)-\Delta_{21}\left(F_{T}\right)\left(\beta_{0}-b\left(F_{T}\right)\right)$ a.s.,

2. $\quad \hat{\alpha}_{L I M L} \rightarrow \alpha_{0}+\Delta_{11}\left(F_{T}\right)+\Pi_{21} b\left(F_{T}\right)-\Delta_{21}\left(F_{T}\right)\left(\beta_{0}-b\left(F_{T}\right)\right)$ a.s.

\section{Tests of Hypothesis}

In order to perform tests on the slope parameters $\beta$, we need to be able to estimate $A\left(F_{T}\right)$ consistently. We focus on the case where the factor loadings in the reduced form explanatory variables and the reduced form errors are uncorrelated conditional on $\mathcal{F}$. In the development of the above asymptotic results, we have shown that conditional on $\mathcal{F}, \hat{\Pi}_{22} \rightarrow \Pi_{22}$ a.s., $\hat{H} \rightarrow H\left(F_{T}\right)$ a.s., $\hat{\beta}_{T S L S} \rightarrow \beta_{0}$ a.s., $\hat{\beta}_{\text {LIML }} \rightarrow \beta_{0}$ a.s., and

$$
\hat{Q}^{*}=\frac{1}{N} \sum_{i=1}^{N}\left(\begin{array}{cc}
\left(w_{i}-\bar{w}\right)^{\prime}\left(w_{i}-\bar{w}\right) & \left(w_{i}-\bar{w}\right)^{\prime}\left(x_{i}-\bar{x}\right) \\
\left(x_{i}-\bar{x}\right)^{\prime}\left(w_{i}-\bar{w}\right) & \left(x_{i}-\bar{x}\right)^{\prime}\left(x_{i}-\bar{x}\right)
\end{array}\right) \rightarrow Q^{*}\left(F_{T}\right) \text { a.s. }
$$

Lemma 3. Let $\hat{\Theta}=\frac{1}{N} \sum_{i=1}^{N} \operatorname{vec}\left[\left(z_{i}-\bar{z}\right)^{\prime} \hat{e}_{i}\right] \operatorname{vec}\left[\left(z_{i}-\bar{z}\right)^{\prime} \hat{e}_{i}\right]^{\prime}$, where $\hat{e}_{i}=y_{i}-\left(\hat{\pi}_{10}, \hat{\Pi}_{20}\right)-z_{i} \hat{\Pi}$. Given Assumption 1 i-vi, conditional on $\mathcal{F}, \hat{\Theta} \rightarrow \Theta\left(F_{T}\right)$ a.s. 
Therefore, let $\hat{\beta}$ be either the panel TSLS or LIML estimator. Then, conditional on $\mathcal{F}$,

$$
\hat{A}=\left(\Pi_{22} \hat{H}^{\prime} \hat{\Pi}_{22}\right)^{-1} \hat{\Pi}_{22}^{\prime} \hat{H}\left(\left(1,-\hat{\beta}^{\prime}\right) \otimes\left(\left(0, I_{k_{2}}\right)\left(S^{\prime} \hat{Q}^{*} S\right)^{-1}\right) S^{\prime}\right) \hat{\Theta}^{\frac{1}{2}} \rightarrow A\left(F_{T}\right)
$$

a.s., and

$$
\hat{A}^{*}=\left(\left(1,-\hat{\beta}^{\prime}\right) \otimes\left(\left(\left(I_{k_{1}}, 0\right)-\hat{\Pi}_{21}\left(\hat{\Pi}_{22}^{\prime} \hat{H} \hat{\Pi}_{22}\right)^{-1} \hat{\Pi}_{22}^{\prime} \hat{H}\left(0, I_{k_{2}}\right)\right)\left(S^{\prime} \hat{Q}^{*} S\right)^{-1}\right) S^{\prime}\right) \hat{\Theta}^{\frac{1}{2}} \rightarrow A^{*}\left(F_{T}\right)
$$

a.s.

Then, it is straightforward to obtain the following result.

Theorem 5. If Assumptions $1 i-v i$ and 2 hold and $R$ is a known $q \times p$ matrix of rank $q<p$ and $r$ is a known and fixed $q \times 1$ vector, such that $R \beta_{0}=r$, then,

1. $\sqrt{N}\left(R \hat{A} \hat{A}^{\prime} R^{\prime}\right)^{\frac{1}{2}}\left(R \hat{\beta}_{T S L S}-r\right) \rightarrow^{D} N\left(0, I_{q}\right)$,

2. $\sqrt{N}\left(R \hat{A} \hat{A}^{\prime} R^{\prime}\right)^{\frac{1}{2}}\left(R \hat{\beta}_{\text {LIML }}-r\right) \rightarrow^{D} N\left(0, I_{q}\right)$.

The above theorem allows us to construct $t$ and $F$ tests on $\beta_{0}$ having standard asymptotic distributions under the null hypothesis. Test on the coefficients of the exogenous variables in the structural equation can be constructed using standard procedures.

Theorem 6. Assumptions $1 i$-vi and 2 hold and $R^{*}$ is a known $q \times k_{1}$ matrix of rank $q<k_{1}$ and $r^{*}$ is a known and fixed $q \times 1$ vector, such that $R^{*} \alpha_{0}=r^{*}$ then,

1. $\sqrt{N}\left(R \hat{A}^{*} \hat{A}^{*^{\prime}} R^{\prime}\right)^{\frac{1}{2}}\left(R^{*} \hat{\alpha}_{T S L S}-r^{*}\right) \rightarrow^{D} N\left(0, I_{q}\right)$,

2. $\sqrt{N}\left(R \hat{A}^{*} \hat{A}^{*^{\prime}} R^{\prime}\right)^{\frac{1}{2}}\left(R^{*} \hat{\alpha}_{L I M L}-r^{*}\right) \rightarrow^{D} N\left(0, I_{q}\right)$.

\section{Conclusions}

The paper has studied the effects of common shocks on the panel TSLS and LIML estimators. It has shown that the panel TSLS and LIML estimators are consistent and have asymptotic covariance-matrix mixed-normal distributions when the factor loadings in the reduced form explanatory variables and the reduced form errors are uncorrelated conditional on the common shocks. However, neither the panel TSLS nor the panel LIML estimator is consistent when this is not the case. The paper has also shown that tests on the structural parameters have standard asymptotic distributions under the null hypothesis when the factor loadings in the errors and the regressors are uncorrelated given the common shocks. These results extend those of Andrews (2005) to model with classical endogeneity, and suggest that accounting for classical endogenity does not address the problem of factors endogeneity.

Supplementary Materials: Proofs of all results are available online at http:/ / www.mdpi.com/2225-1146/6/2/ 19/s1.

Acknowledgments: This research was partially supported by Australian Research Council grant DP0985432.

Author Contributions: The authors contributed jointly to the paper.

Conflicts of Interest: The authors declare no conflict of interest.

\section{References}

Ahn, Seung Chan, Young Hoon Lee, and Peter Schmidt. 2001. Gmm estimation of linear panel data models with time-varying individual effects. Journal of Econometrics 102: 219-55.

Ahn, Seung C., Young H. Lee, and Peter Schmidt. 2013. Panel data models with multiple time-varying individual effects. Journal of Econometrics 174: 1-14. 
Alonso-Borrego, Cesar, and Manuel Arellano. 1999. Symmetrically normalized instrumental-variable estimation using panel data. Journal of Business \& Economic Statistics 17: 36-49.

Andrews, Donald W. K. 2005. Cross-section regression with common shocks. Econometrica 73: 1551-85.

Arellano, Manuel. 2016. Modelling optimal instrumental variables for dynamic panel data models. Research in Economics 70: 238-61.

Bai, Jushan. 2009. Panel data models with interactive fixed effects. Econometrica 77: 1229-79.

Cabrera, Manuel Ordóñez, Andrew Rosalsky, and Andrei Volodin. 2012. Some theorems on conditional mean convergence and conditional almost sure convergence for randomly weighted sums of dependent random variables. Test 21: 369-85.

Dedecker, Jérôme, and Florence Merlevède. 2002. Necessary and sufficient conditions for the conditional central limit theorem. Annals of Probability 30: 1044-81.

Grzenda, Wioletta, and Wieslaw Zięba. 2008. Conditional central limit theorem. International Mathematical Forum 31: 1521-28.

Harding, Matthew, and Carlos Lamarche. 2011. Least squares estimation of a panel data model with multifactor error structure and endogenous covariates. Economics Letters 111: 197-99.

Harding, Matthew, and Carlos Lamarche. 2014. Estimating and testing a quantile regression model with interactive effects. Journal of Econometrics 178: 101-13.

Hausman, Jerry A. 1983. Specification and estimation of simultaneous equation models. In Handbook of Econometrics. Edited by Z. Griliches and M. D. Intriligator. Amsterdam: North-Holland Publishing Company, vol. 1, pp. 391-448.

Kuersteiner, Guido M., and Ingmar R. Prucha. 2013. Limit theory for panel data models with cross sectional dependence and sequential exogeneity. Journal of Econometrics 174: 107-26.

Majerek, Dariusz, Wioletta Nowak, and Wieslaw Zięba. 2005. Conditional strong law of large numbers. International Journal of Pure and Applied Mathematics 20: 143-56.

Pesaran, M. Hashem. 2006. Estimation and inference in large heterogeneous panels with a multifactor error structure. Econometrica 74: 967-1012.

Phillips, Peter C. 1988. Conditional and unconditional statistical independence. Journal of Econometrics 38: 341-48.

Rao, B. L. S. Prakasa. 2009. Conditional independence, conditional mixing and conditional association. Annals of the Institute of Statistical Mathematics 61: 441-60.

Robertson, Donald, and Vasilis Sarafidis. 2015. Iv estimation of panels with factor residuals. Journal of Econometrics 185: 526-41.

Roussas, George G. 2008. On conditional independence, mixing, and association. Stochastic Analysis and Applications 26: 1274-309.

Schmidt, Peter. 1976. Econometrics. New York: M. Dekker.

Wansbeek, Tom, and Erik Meijer. 2000. Measurement Error and Latent Variables in Econometrics. New York: North Holland.

Wansbeek, Tom, and Dennis Prak. 2017. LIML in the static linear panel data model. Econometric Reviews 36: 385-95. Wooldridge, Jeffrey M. 2005. Instrumental variables estimation with panel data. Econometric Theory 21: 865-69.

Yuan, De-Mei, Li-Ran Wei, and Lan Lei. 2014. Conditional central limit theorems for a sequence of conditional independent random variables. Journal of the Korean Mathematical Society 51: 1-15.

(C) 2018 by the authors. Licensee MDPI, Basel, Switzerland. This article is an open access article distributed under the terms and conditions of the Creative Commons Attribution (CC BY) license (http:/ / creativecommons.org/licenses/by/4.0/). 